\title{
Tumor Response Criteria in Oncoimaging: RECIST Criteria and beyond-Part 1
}

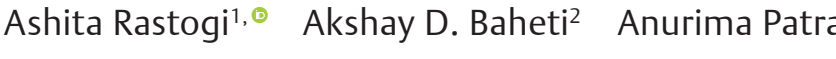 \\ ${ }^{1}$ Department of Radiodiagnosis, Delhi State Cancer Institute, \\ New Delhi, India \\ 2Department of Radiodiagnosis, Tata Memorial Hospital, Mumbai, \\ Maharashtra, India \\ ${ }^{3}$ Department of Imaging, Dana Farber Cancer Institute, Harvard \\ Medical School, Boston, Massachusetts, United States \\ 4Department of Radiology, Brigham and Women's Hospital, \\ Harvard Medical School, Boston, Massachusetts, United States
}

\author{
Sree Harsha Tirumani ${ }^{3,4}$
}

J Gastrointestinal Abdominal Radiol ISGAR 2019;2:98-106

\begin{abstract}
Keywords

- MD Anderson criteria bone

- oncologic imaging

- RECIST

- tumor response

The rapid recent advances in oncology have made the dream of precision oncology a reality, with targeted therapy available for various tumors depending on the molecular genotype. This has led to the corresponding development of personalized radiology as well, with various tumor response criteria used to characterize disease response/ progression depending on chemotherapy used. In these two review articles, we review the various tumor response criteria widely applied in both research and clinical settings. These include the classic size-based criteria such as RECIST (Response Evaluation Criteria in Solid Tumors) 1.1 and the WHO (World Health Organization) criteria, as also various other criteria such as Choi and modified Choi criteria for tumors treated by targeted therapy, EASL and modified RECIST (mRECIST) criteria for hepatocellular carcinomas, and immune-related response criteria (irRC) and immune RECIST (iRECIST) for patients on immunotherapy. Other clinically important criteria such as PERCIST (PET Response Criteria In Solid Tumors) for positron emission tomography-computed tomography (PET-CT) and the MD Anderson criteria for evaluating bone metastases are also highlighted.
\end{abstract}

\begin{abstract}
Address for correspondence Akshay D. Baheti, MD, Department of Radiodiagnosis, Tata Memorial Hospital, Dr. E. Borges Road, Parel, Mumbai 400012, Maharashtra, India (e-mail: akshaybaheti@gmail.com).
\end{abstract}

\section{Introduction}

Cancer is among the most common cause of deaths worldwide, with enormous research going on into its pathogenesis and treatment and numerous novel therapies being developed and approved every year. These new therapies need to go through multiple preliminary trials to ascertain their safety and efficacy before being approved. The gold standard to prove drug efficacy is to prove improved survival, but this is usually a very long process. Radiologic assessment of tumor size and morphology thus proves as an extremely useful surrogate marker for quick assessment of response (or the lack of it) to a new treatment and is now one of the methods of assessment of choice in most clinical trials to assess drug efficacy. Over time, received

March 3, 2019

accepted

March 26, 2019

published online

July 22, 2019 well-defined response assessment criteria have developed to ensure objective and standardized assessment of tumor response or progression.

RECIST (Response Evaluation Criteria in Solid Tumors) criteria, introduced in 2000 and updated (RECIST 1.1) in 2009, is the workhorse for response assessment in most clinical drug trials. ${ }^{1,2}$ However, RECIST criteria predominantly remain limited to size for now, while, with the evolution of newer novel cancer treatments such as targeted therapy and immunotherapy, tumors may respond in different ways beyond a conventional decrease in size (such as with decreased enhancement). Similarly, tumors such as hepatocellular carcinoma or sites such as bone metastases or a primary brain neoplasm may also respond in different ways to treatment. Thus, multiple alternate response criteria
C2019 Indian Society of Gastrointestinal and Abdominal Radiology
License terms

() (1) $\ominus \circledast$ 10.1055/s-0039-1692021 ISSN 2581-9933. 
also exist to be used in specific situations. ${ }^{3-5}$ It is important to realize that criteria such as RECIST are created specifically to be used in scientific trials and should not be stringently applied in routine clinical practice. However, the principles behind these criteria can and indeed must be used while reporting every patient on cancer therapy. In these two review articles, we give a historical perspective on the emergence of tumor response criteria, review the salient points and shortcomings of RECIST and various alternate response criteria, and focus on the various principles behind these criteria that every radiologist must be aware of in routine clinical practice. Part I focuses on RECIST criteria, whereas the alternate response criteria are discussed in part II.

\section{World Health Organization Criteria}

The first attempt to standardize the assessment and reporting of outcomes of therapy in clinical trials world over was made by the World Health Organization (WHO), in conjunction with multiple other relevant international organizations. They established the WHO criteria in $1981^{6,7}$ for clinical, radiologic, and other measurements of tumor response. The WHO guidelines encompassed all aspects of cancer diagnosis and management, including the data collection, reporting the type of treatment, response, toxicity, results, etc. ${ }^{6}$

These guidelines required bidimensional measurements (longest overall diameter and longest diameter perpendicular to it) to be taken and the surface area to be recorded as the sum of the products of diameters. The sum of the surface areas thus determined for multiple measurable lesions of single/multiple organ sites was then used to assess response. A minimum of 4 weeks interval was recommended between two observations to assess response, which could be assessed clinically, radiologically, biochemically, or with surgical-pathological staging. Decrease in the surface area by $>50 \%$ was considered partial response (PR) ( - Table 1 ). ${ }^{6,7}$ This cutoff was based on a study involving 16 oncologists that demonstrated that there was only a 6.8 to $7.8 \%$ error in assessing response with a cutoff of $50 \%$ decrease in surface area, whereas the error rate was as high as 19 to $25 \%$ if the cutoff was kept at $25 \% .{ }^{8}$ Progression, on the other hand, was defined as $>25 \%$ increase in the surface area of the tumor.

\section{RECIST criteria}

The WHO criteria had several shortcomings, as there was no clear definition regarding the number and size of lesions, which should be measured, and using the sum of products of diameters was a tedious process. ${ }^{1,9}$ As a result, an international taskforce was established to formulate more specific and simpler guidelines. This taskforce established the RECIST guidelines in $2000,{ }^{1}$ which specified the techniques, modalities, and criteria to assess response (or the lack of it) to treatment. - Table 1 compares the WHO criteria with RECIST criteria.

RECIST did away with bidimensional measurements based on a study on 569 patients, which concluded that unidimensional measurement of the maximum tumor
Table 1 WHO criteria and RECIST 1.1 criteria for response assessment

\begin{tabular}{|c|c|c|}
\hline $\begin{array}{l}\text { Response } \\
\text { categories }\end{array}$ & WHO criteria & RECIST 1.1 \\
\hline CR & $\begin{array}{l}\text { Disappear- } \\
\text { ance of } \\
\text { all known } \\
\text { disease for at } \\
\text { least } 4 \mathrm{wk}\end{array}$ & $\begin{array}{l}\text { Disappearance of all target } \\
\text { lesions (including reduction } \\
\text { in short-axis diameter of } \\
\text { lymph nodes to }<10 \mathrm{~mm} \text { ) } \\
\text { Disappearance of all nontar- } \\
\text { get lesions and normalization } \\
\text { of tumor marker level }\end{array}$ \\
\hline PR & $\begin{array}{l}\geq 50 \% \\
\text { decrease } \\
\text { in the total } \\
\text { tumor size of } \\
\text { lesions at two } \\
\text { observations } \\
\text { at least } 4 \text { wk } \\
\text { apart }\end{array}$ & $\begin{array}{l}\geq 30 \% \text { decrease in the sum } \\
\text { of maximum diameters of } \\
\text { target lesions from baseline } \\
\text { Persistence of one or more } \\
\text { nontarget lesions and/or } \\
\text { the maintenance of tumor } \\
\text { marker level above the nor- } \\
\text { mal limits }\end{array}$ \\
\hline PD & $\begin{array}{l}\geq 25 \% \\
\text { increase in } \\
\text { size of one } \\
\text { or more } \\
\text { measurable } \\
\text { lesions or ap- } \\
\text { pearance of } \\
\text { new lesions }\end{array}$ & $\begin{array}{l}\geq 20 \% \text { increase in the sum } \\
\text { of maximum diameters of } \\
\text { target lesions from nadir (i.e., } \\
\text { from when the smallest sum } \\
\text { of diameters is achieved) or } \\
\text { appearance of one or more } \\
\text { new lesions } \\
\text { Unequivocal progression of } \\
\text { existing nontarget lesions or } \\
\text { appearance of one or more } \\
\text { new sites of disease }\end{array}$ \\
\hline SD & $\begin{array}{l}\text { Neither } \\
a \geq 50 \% \\
\text { decrease } \\
\text { nor } a \geq 25 \% \\
\text { increase in } \\
\text { lesions' size } \\
\text { has been } \\
\text { demonstrated }\end{array}$ & Neither PR or PD \\
\hline
\end{tabular}

Abbreviations: $C R$, complete response; $P D$, progressive disease; $P R$, partial response; RECIST, Response Evaluation Criteria in Solid Tumors; SD, stable disease; WHO, World Health Organization.

diameter is sufficient to assess tumor response. ${ }^{10}$ It defined the imaging parameters (particularly for spiral computed tomography [CT]) to be used for imaging cancer patients in trials, the minimum size of measurable lesions, the use of single largest diameter for assessing response, and the new cutoffs based on the sum of largest diameters. The cutoff for PR was changed to 30\% from 50\% (as a 30\% decrease in diameter would be equivalent to a $50 \%$ decrease in area).

One of the important implications of adapting RECIST over WHO criteria was the change in definition of progressive disease (PD). It was realized that the stringent WHO cutoff was leading to the early removal of patients from trial, who could have eventually benefited from therapy due to delayed tumor response. The cutoff was hence increased to enable more patients to remain in the trial. RECIST defined tumor progression as a $20 \%$ increase in the longest diameter (which translates into $73 \%$ increase in the tumor volume), as compared with the WHO cutoff of $25 \%$ increase in surface area (which translates into $40 \%$ increase in volume). ${ }^{11}$ 


\section{RECIST 1.1 criteria}

The RECIST criteria were quickly adopted by the regulatory authorities and pharmaceutical industry. Over a few years, however, certain shortcomings were noted such as in the assessment of lymph nodes and on the use of newer techniques such as multiple detector CT (MDCT), magnetic resonance imaging (MRI), and positron emission tomography-computed tomography (PET-CT). Accordingly, RECIST version 1.1 was published in 2009 to address these limitations, predominantly based on the review of a large database of $>6,500$ patients (-Table 2)..$^{12}$ The RECIST 1.1 guidelines ${ }^{13,14}$ are discussed in detail ahead.

\section{Specifications for Imaging}

1. The RECIST working group gives recommendations for standardization of imaging in clinical trials in RECIST 1.1. Emerging tools such as functional imaging techniques or volumetric assessment are not included. It must be noted that superficial lesions, at least $10 \mathrm{~mm}$ in size, can be

Table 2 Changes from RECIST 1.0 to RECIST $1.1^{13,14}$

\begin{tabular}{|c|c|c|}
\hline Criteria & RECIST 1.0 & RECIST 1.1 \\
\hline $\begin{array}{l}\text { Lymph } \\
\text { nodes }\end{array}$ & Not specified & $\begin{array}{l}\text { Incorporated; short-axis } \\
\text { diameter to be measured } \\
\text { and categorized as follows: } \\
\geq 15 \mathrm{~mm} \text { are measurable } \\
\text { and may be considered } \\
\text { target lesions } \\
10-15 \mathrm{~m} \text { nontarget lesions } \\
<10 \mathrm{~m} \text { nonpathologic } \\
\text { lesions }\end{array}$ \\
\hline $\begin{array}{l}\text { Number } \\
\text { of lesions } \\
\text { measured }\end{array}$ & $\begin{array}{l}10 \text { lesions }(\leq 5 \text { in } \\
\text { any one organ) }\end{array}$ & $\begin{array}{l}\text { Five lesions ( } \leq 2 \text { in any one } \\
\text { organ) }\end{array}$ \\
\hline $\begin{array}{l}\text { Definition } \\
\text { of pro- } \\
\text { gressive } \\
\text { disease }\end{array}$ & $\begin{array}{l}20 \% \text { increase in } \\
\text { sum of longest } \\
\text { diameter or } \\
\text { new lesions, } \\
\text { unequivocal } \\
\text { progression } \\
\text { considered to } \\
\text { indicate pro- } \\
\text { gressive disease }\end{array}$ & $\begin{array}{l}20 \% \text { increase in sum } \\
\text { of longest diameter; } \\
\geq 5 \mathrm{~mm} \text { increase in size; } \\
\text { new lesions; detailed } \\
\text { description of unequivocal } \\
\text { progression }\end{array}$ \\
\hline $\begin{array}{l}\text { New } \\
\text { lesions }\end{array}$ & $\begin{array}{l}\text { No } \\
\text { specifications }\end{array}$ & $\begin{array}{l}\text { Provides guidelines for } \\
\text { new lesions to qualify for } \\
\text { progressive disease }\end{array}$ \\
\hline $\begin{array}{l}\text { Specifica- } \\
\text { tions for } \\
\text { imaging }\end{array}$ & Limited & $\begin{array}{l}\text { Updated to include MRI } \\
\text { and PET-CT }\end{array}$ \\
\hline $\begin{array}{l}\text { Overall } \\
\text { response }\end{array}$ & $\begin{array}{l}\text { Table-inte- } \\
\text { grated target } \\
\text { and nontarget } \\
\text { lesions }\end{array}$ & $\begin{array}{l}\text { Additional table with non- } \\
\text { target lesion only } \\
\text { Guidance on CR in face of } \\
\text { residual tissue }\end{array}$ \\
\hline $\begin{array}{l}\text { Confir- } \\
\text { mation of } \\
\text { response }\end{array}$ & $\begin{array}{l}\text { For } C R \text { and } P R, \\
\text { criteria must } \\
\text { be met again } \\
4 \text { wk after initial } \\
\text { documentation }\end{array}$ & $\begin{array}{l}\text { Required only for non- } \\
\text { randomized trials with } \\
\text { primary endpoint of } \\
\text { response }\end{array}$ \\
\hline
\end{tabular}

Abbreviations: $\mathrm{CR}$, complete response; $\mathrm{MRI}$, magnetic resonance imaging; $\mathrm{PET}-\mathrm{CT}$, positron emission tomography-computed tomography; PR, partial response; RECIST, Response Evaluation Criteria in Solid Tumors; WHO, World Health Organization. evaluated by physical examination alone. However, even in these cases, it is preferable to have at least one lesion measurable on $\mathrm{CT}$, if possible.

2. The CT slice thickness for assessing patients using RECIST criteria should be no greater than $5 \mathrm{~mm}$. As a general rule, the minimum size of a measurable lesion at baseline should not be less than double the slice thickness. Hence, the minimum measurable lesion size on CT has been set at $10 \mathrm{~mm}$ to avoid discrepancies from partial volume averaging and for accurate and reproducible measurement.

3. Measurement of lesions on a chest radiograph is possible and permissible when they are surrounded by normal lung parenchyma. However, a CT should be preferred over radiographs for measurement of pulmonary and mediastinal lesions as well as for identification of new lesions.

4. CT scans, when performed, should be contiguous throughout the anatomic region of interest and a CT of the chest, abdomen, and pelvis suffices for most tumors to include potential sites of metastases. Adequate coverage of these sites is important because any lesion identified at a later date in an area not previously scanned would be considered as "new lesion." Additionally, based on signs and symptoms of the patient, other areas may be scanned at baseline. Most solid tumors may be scanned with postcontrast CT in a single phase, whereas triphasic $\mathrm{CT}$ is recommended for hepatocellular cancers and neuroendocrine tumors for optimal visualization. In case of known contrast allergy or renal insufficiency, noncontrast CT or MRI should be performed at baseline depending on the tumor type and anatomic location. Whereas organ-specific contrast agents such as those for liver lesions may be used in patient care, their use in clinical trials is not recommended as yet.

5. Ultrasound examination has not been endorsed by RECIST working group for use in trials due to subjectivity of measurement and operator dependency. Similarly, skeletal scintigraphy is not incorporated in the RECIST guidelines.

6. No clear-cut guidelines are available on the technical use of MRI in clinical trials owing to image acquisition variables that differ from scanner to scanner and also due to the inconstant availability of MRI scanners at different institutions.

\section{Definitions}

- Measurable lesion: A lesion that has the longest diameter of $\geq 10 \mathrm{~mm}$ on CT or clinically or $\geq 20 \mathrm{~mm}$ on a chest radiograph is considered as "measurable." Lymph nodes on CT are considered as a measurable lesion if the short-axis diameter is $\geq 15 \mathrm{~mm}$. Skeletal metastasis is considered a measurable lesion if it has a soft tissue component $\geq 10 \mathrm{~mm}$ on CT.

- Nonmeasurable lesion: This category includes lesions that do not meet the criteria of measurable lesions, such as small lesions with a longest diameter of $<10 \mathrm{~mm}$, skeletal metastases without a soft tissue component, ascites, pleural effusion, lymphangitic spread of tumor, leptomeningeal disease, inflammatory breast disease, and a palpable abdominal mass not confirmed by imaging. Lymph nodes between 10 and $15 \mathrm{~mm}$ in short-axis diameter are also considered as pathologically enlarged but not measurable. 
- Target lesion: Given that all it would be tedious to measure each and every lesion in a patient with widespread metastatic disease on the baseline scan and on each and every follow-up scan (without really adding further to patient management), the concept of target lesion was introduced by the RECIST working group in the original RECIST criteria. These are appropriately selected lesions on the baseline scan, which will be measured on all subsequent scans to evaluate response. All measurable lesions are candidates for selection as target lesions on the baseline staging scan. A maximum of five target lesions (maximum two sites per organ) should be included on the baseline scan as per RECIST 1.1 guidelines. The baseline sum of longest diameters of these five lesions is then calculated.

\section{How to Measure Lesions Using RECIST 1.1 Criteria}

1. RECIST 1.1 assumes most lesions to be more or less spherical in shape. Hence, the measurement for almost all lesions is to be performed in the axial plane with the exception of certain tumors such as paraspinal lesions that are better measured in coronal or sagittal plane. At follow-up, the maximum diameter of the lesion must be measured even if it is now visualized at a different slice level or in a different orientation as compared with the baseline imaging ( - Fig. $\mathbf{1}$ ).

2. For lesions that separate or coalesce at follow-up, the longest diameter of the target lesion includes the maximum diameters of the fragmented portions added together in case of one lesion separating into two or more lesions. If two separate lesions become confluent, the sum of the longest diameters should be measured as the longest diameter ( - Fig. 2).

3. If the lesion becomes too small and indistinct to measure (but has not completely disappeared) on follow-up imaging, a default value of $5 \mathrm{~mm}$ is assigned to the lesion for measurement purposes (derived from the slice thickness of $5 \mathrm{~mm}$ on $\mathrm{CT}$ ). On the other hand, the measurement is recorded as $0 \mathrm{~mm}$ if the lesion disappears completely.

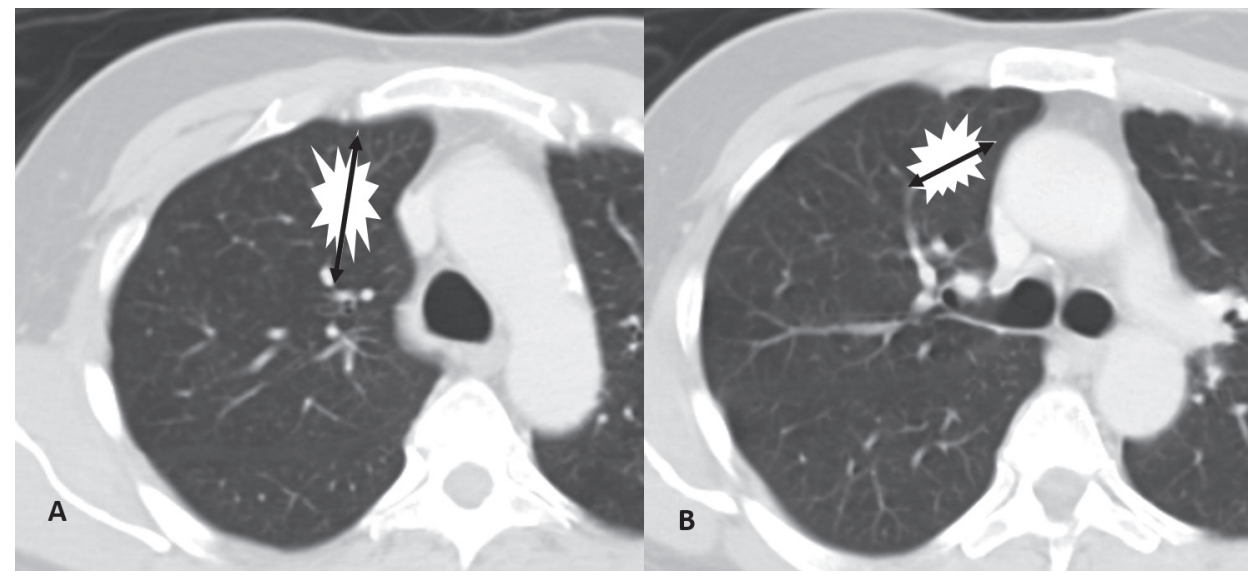

Fig. 1 Diagrammatic representation of a lung lesion at baseline (A) and at follow-up (B). At follow-up, the lesion has decreased in size. The longest diameter of the lesion is measured in accordance with RECIST guidelines as shown in the figure at a different axis (vector angle) than baseline and also at a different slice level $[(\mathbf{A})$ is at the level of arch of aorta and $(\mathbf{B})$ is at the level of tracheal bifurcation].

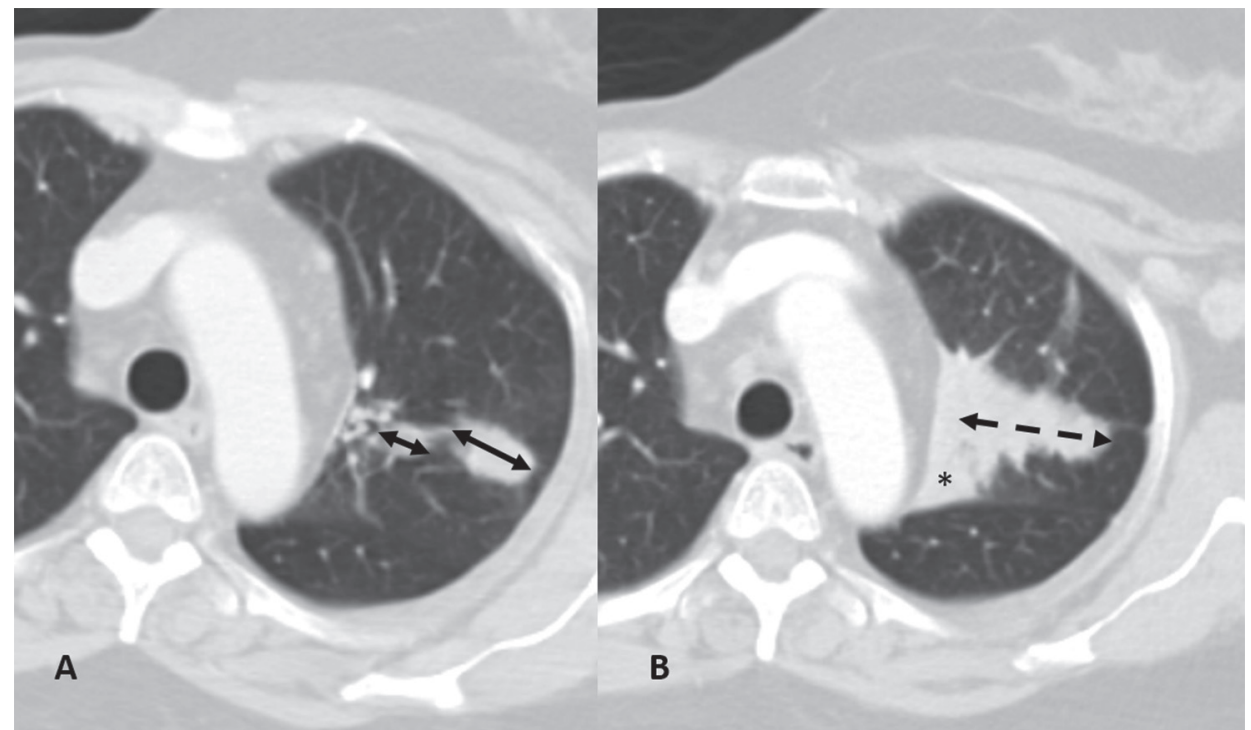

Fig. 2 Lung metastases in a patient with carcinoma of the right breast. (A) Two lesions in left upper lobe were selected as target lesions at baseline. (B) The lesions coalesced at follow-up after 8 weeks to form a single lesion. The longest diameter in (B) exceeded $20 \%$ of baseline sum of longest diameters of the two lesions in (A), consistent with progressive disease. Also seen is associated subsegmental collapse (asterisk). 
4. Specifications have been provided for bone lesions, cystic lesions, as well as for previously treated lesions in the revised guidelines. Purely osteoblastic lesions are considered as nonmeasurable whereas only lytic or mixed lytic-blasting bone lesions with measurable soft tissue component on CT or MRI can be considered as measurable lesions. Cystic lesions that are probably metastatic may be considered as target lesions if measurable, preferably only in the absence of other noncystic target lesions. Tumor lesions in a previously irradiated area are not considered measurable unless they show unequivocal progression.

5. It must be noted that the original RECIST criteria included up to 10 target lesions. The RECIST working group subsequently analyzed data from 16 trials conducted between 1993 and 2005 to compare response and progression when measuring 5 target lesions compared with 10 target lesions. ${ }^{12}$ They observed no significant difference in the overall response rate, and hence the number of target lesions was reduced to five in RECIST 1.1, thus simplifying response assessment.

\section{Assessment of Tumor Burden and Target Lesions}

To assess tumor response, it is essential to objectively estimate the overall tumor burden (sum of longest diameters) at baseline (pretreatment) and use this to compare on subsequent studies. ${ }^{2}$ Target lesions can be selected from among all measurable lesions based on their size, organ of involvement, and, importantly, potential for reproducible accurate measurements on subsequent scans. For example, a primary tumor arising from a hollow viscous such as carcinoma stomach should not be considered as a target lesion, as its measurement would vary based on the degree of distention of the stomach and its orientation ( - Fig. 3 ). It is acceptable to instead measure the largest reproducible lesion (metastatic node or liver metastasis) in such cases. Similarly, an irregular shaped or an ill-defined lesion should be avoided, if possible, as reproducible measurements may be difficult.

Other sites of disease (including pathologic nodes and nonmeasurable disease) should also be documented at baseline. Measurements are not required for them; they can be documented as either "present," "absent," or rarely as "unequivocal progression." Nor does each and every lesion need an individual mention; for example, they can be mentioned as "multiple enlarged retroperitoneal nodes" or "multiple pulmonary metastases."

Given these criteria, only patients with measurable disease can be included in trials where objective tumor response is the primary endpoint. On the other hand, trials that utilize tumor progression as the endpoint may include patients with nonmeasurable disease as well if so specified in the trial protocol.

\section{Response Evaluation}

Tumor response can be classified as complete response (CR), PR, stable disease (SD), or PD (-Table 1). It is evaluated based on the percentage change in the sum of diameters of target lesions in the restaging scan as compared with the

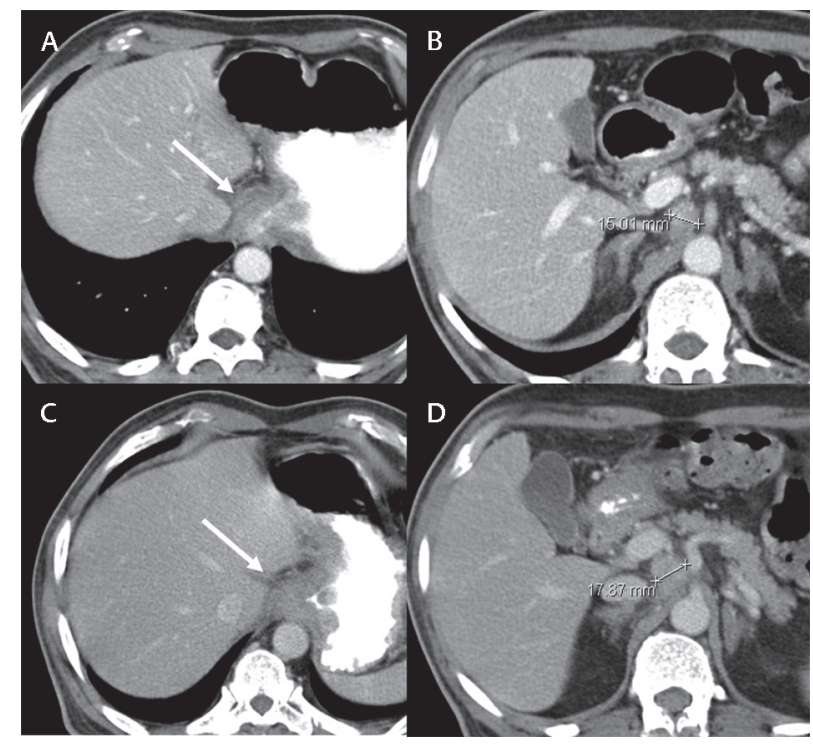

Fig. 3 The patient with carcinoma stomach. Baseline contrastenhanced computed tomography (CT) (A, B) shows wall thickening at the gastroesophageal junction (arrow in $\mathbf{A}$ ) and an enlarged aortocaval node measuring $15 \mathrm{~mm}$ in short axis (B). Postchemotherapy CT (C, D) shows an apparent increase in the gastroesophageal junction thickening (arrow in C), which is difficult to assess as the stomach is less distended compared with (A) and reflects inadequacy of RECIST. The aortocaval node that was considered as one of the target lesions now measures $18 \mathrm{~mm}$ in short axis (B) and overall disease is stable by RECIST criteria.

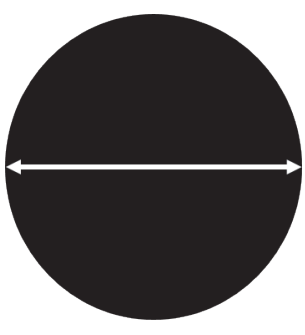

A. Baseline Scan

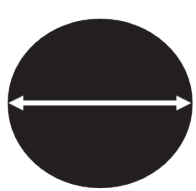

B. First follow-up

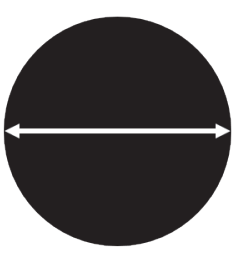

C. Second follow-up

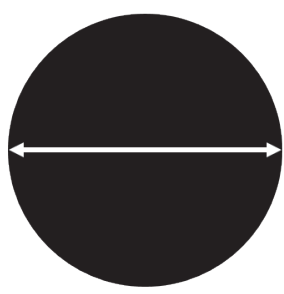

D. Third follow-up

Fig. 4 Response evaluation using RECIST criteria in a well-defined 20-mm target lesion (A) at baseline scan. (B) On first follow-up scan after chemotherapy, the target lesion has shrunk to $10 \mathrm{~mm}$ ( $50 \%$ decrease), consistent with partial response. (C) On second follow-up scan, the target lesion has increased to $14 \mathrm{~mm}$ ( $40 \%$ increase from the nadir). However, the disease is considered stable as the absolute increase in size is $<5 \mathrm{~mm}$. (D) On third follow-up scan, the lesion has increased further to $18 \mathrm{~mm}$ ( $80 \%$ increase from nadir and $>5 \mathrm{~mm}$ absolute increase) and qualifies for disease progression. 
baseline scan/nadir ( - Fig. 4). Frequency of tumor evaluation is protocol specific, whereas the baseline scan needs to be performed at 4 weeks or, in some cases, only 2 weeks prior to start of therapy, follow-up evaluation may be performed at 6 to 8 weeks probably timed to coincide with end of a chemotherapy cycle.

Response assessment categories have essentially remained unchanged between RECIST to RECIST 1.1. Certain clarifications are listed as follows:

1. Target lesion progression: To qualify for progression, an absolute increase of at least $5 \mathrm{~mm}$ in the longest diameter is required for an individual target lesion in addition to $20 \%$ increase in sum of maximum diameters of target disease (-Fig. 4 ). This helps avoid variability in measurement especially in small-volume disease. It must be remembered that progression is based on comparison with the nadir (i.e., the smallest sum of longest diameters) achieved during the course of the entire treatment.

2. Nonmeasurable disease progression: Assigning progression of nonmeasurable disease requires considerable worsening of the tumor burden. An equivalence can be drawn by labeling nonmeasurable disease progression if there are an approximately $73 \%$ increase in overall disease volumes. ${ }^{2}$ Unequivocal progression can be called even in the presence of SD or partial response in the target lesions, or even in the absence of target lesions ( - Fig. 5). Examples of unequivocal progression include increase in ascites or effusion from trace to severe, or lymphangitic carcinomatosis of lung spreading from a single lobe to diffusely involving both lungs. On the other hand, increase in effusion from moderate to severe or a few more prominent sub $5 \mathrm{~mm}$ nodules in a patient with military pulmonary metastases should not be considered as progression.

3. New lesions: Any new lesion identified at follow-up imaging qualifies for disease progression (-Fig. 6). Hence, it is important to determine that the finding of a new lesion is unequivocal and cannot be attributed to a change in scanning technique or imaging modality or does not represent something other than tumor (e.g., posttreatment sclerosis of preexisting bone lesions may render them conspicuous at surveillance imaging). A new lesion especially needs to be viewed with caution if other baseline lesions show partial or CR. If a lesion is visualized at an anatomical location that was not scanned at baseline, it meets the criteria for a new lesion and will indicate disease progression. For a new lesion rendered equivocal because of its size, assessment at subsequent imaging is recommended to confirm

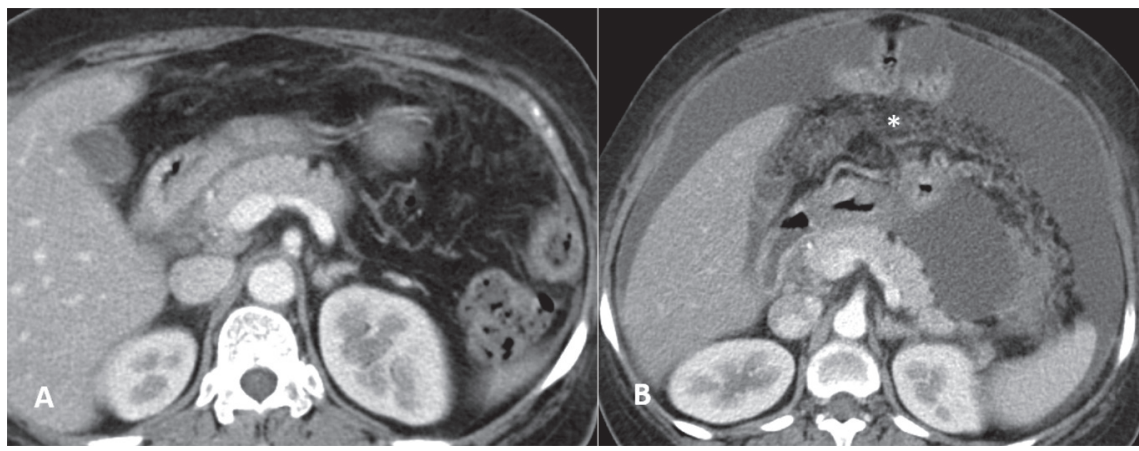

Fig. 5 Nonmeasurable disease progression. The patient with carcinoma stomach with adnexal metastases. Baseline computed tomography (CT) (A) reveals no peritoneal disease. Follow-up CT (B) after chemotherapy reveals severe ascites along with new-onset omental nodularity (asterisk), with no measurable peritoneal lesion. This is consistent with progressive disease due to unequivocal progression of nonmeasurable peritoneal disease.

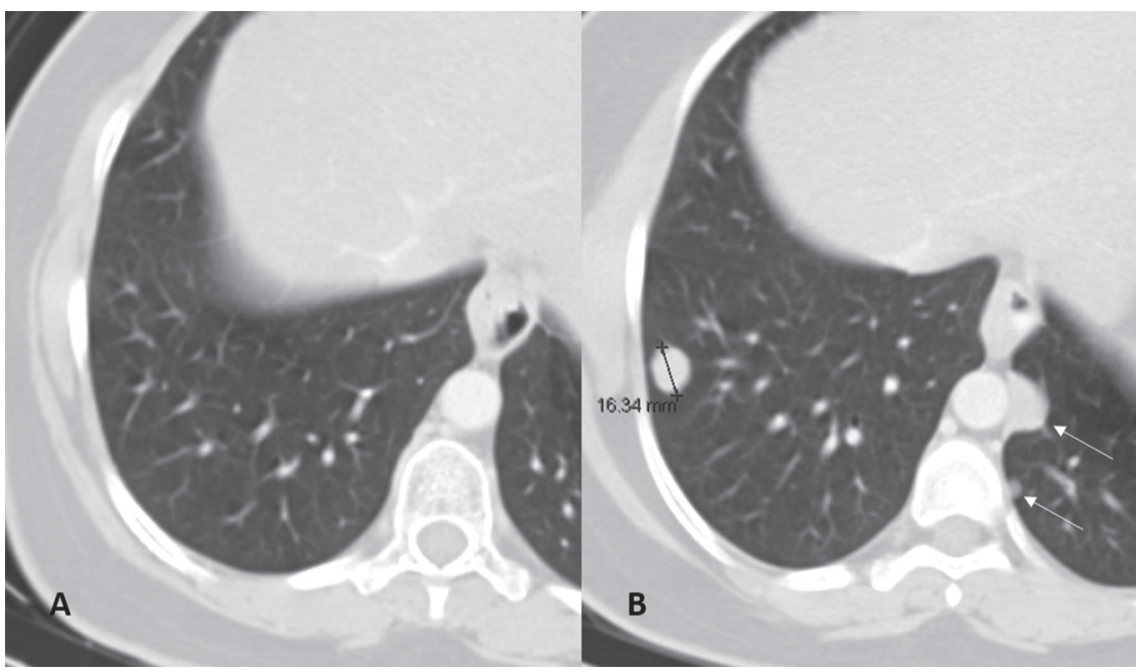

Fig. 6 New lung lesions seen at 12 weeks follow-up (B) consistent with metastases-progressive disease. No lesions were seen in the lung in the previous computed tomography scan (A) in this patient with carcinoma breast. 
the presence of the lesion. If confirmed, disease progression is then declared from the date of the initial scan in such a scenario.

\section{Few Important Points to Note}

1. Assessment of lymph nodes: Evaluation of lymph nodes was inconsistent in the early trials as there were inconsistencies in the size criteria used as also different measurement techniques (long-axis diameter, short-axis diameter, or their ratio). The RECIST working group reassessed 2,747 lymph nodes measured (using two dimensions) as target lesions in 1,235 patients from 16 clinical trials to formulate the guidelines. ${ }^{15}$ Short-axis diameter of lymph node was found to correlate the best with other target lesion measurements and overall disease burden..$^{15} \mathrm{~A}$ cutoff value of $10 \mathrm{~mm}$ for the short-axis diameter was assigned to limit inclusion of normal or minimally enlarged inflamed nodes.

Thus, in RECIST 1.1, lymph nodes are considered pathologically enlarged if $>10 \mathrm{~mm}$ in short-axis diameter. However, nodes between 10 and $15 \mathrm{~mm}$ cannot be included as target lesions (these are counted as nonmeasurable disease), and only those with short-axis diameter $\geq 15 \mathrm{~mm}$ are considered as target lesions ( - Fig. 7 ). It must be noted that lymph nodes

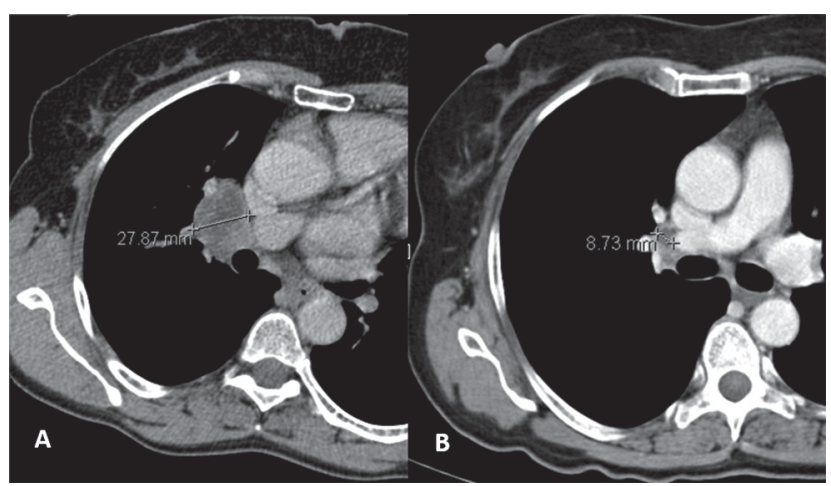

Fig. 7 The patient with carcinoma lung. Baseline scan (A) demonstrates an enlarged necrotic right hilar node with short-axis diameter of $28 \mathrm{~mm}$; this was considered a target lesion. At 3 months follow-up (B), the lymph node decreased in size with the short-axis diameter $<1 \mathrm{~cm}$, consistent with response. Overall, disease was labeled as partial response as there was partial response in other target sites with persistent residual primary lung mass. Please note that if the rest of the disease showed complete resolution, this would be categorized as complete response. are normal anatomical structures and do not disappear completely even after therapy. Hence, nodes can be labeled to demonstrate $\mathrm{CR}$ if the short-axis diameter becomes $<10 \mathrm{~mm}$; however, the measurement of the short-axis diameter still has to be recorded.

2. FDG-PET in assessment: Categorization of new lesions detected on FDG-PET has been incorporated in RECIST 1.1. ${ }^{2}$ In patients with a baseline PET, any new lesion on follow-up PET qualifies for progressive disease. In patients without a baseline PET, any new lesion detected on follow-up PET requires confirmation on CT (at the same time point or at follow-up) to be classified as progressive disease. PERCIST guidelines are discussed in another article.

3. Mesothelioma assessment: Because malignant pleural mesothelioma tends to grow circumferentially than as a sphere, classic uni- or bidimensional measurements are not feasible. RECIST working group proposed measurement of tumor thickness perpendicular to chest wall or mediastinum in two positions at three levels consolidated into a single sum of pleural disease. These modifications correlate with survival and lung function., ${ }^{2,16}$

\section{Potential Pitfalls and Shortcoming}

It is important to safeguard against certain potential pitfalls while using RECIST. Needless to say, it is important to always follow the criteria regarding target lesion selection and measurement stringently. Particularly during a baseline scan, one must ensure that only definitely metastatic lesions are labeled as target lesions and avoid labeling pseudolesions or benign/ indeterminate lesions such as incidental adrenal nodules or hepatic hemangiomas as target lesions ( - Fig. 8).$^{17}$ Review of clinical details such as recent biopsy or surgery may be helpful to avoid inadvertent selection of postoperative seroma or granulation tissue as a target lesion.

In addition, it must be remembered that lesions may appear more or less conspicuous on different phases after contrast administration-particularly in the case of hepatic lesions (- Fig. 9). ${ }^{17}$ For instance, hypervascular neuroendocrine tumor metastases to the liver can appear very different in size and attenuation, depending on the vascular phase of acquisition; comparing an arterial phase sequence on the current study with a venous phase sequence from a prior study may lead to incorrect labeling of tumor response. Hence, care is required to have consistent image acquisition with $\mathrm{CT}$

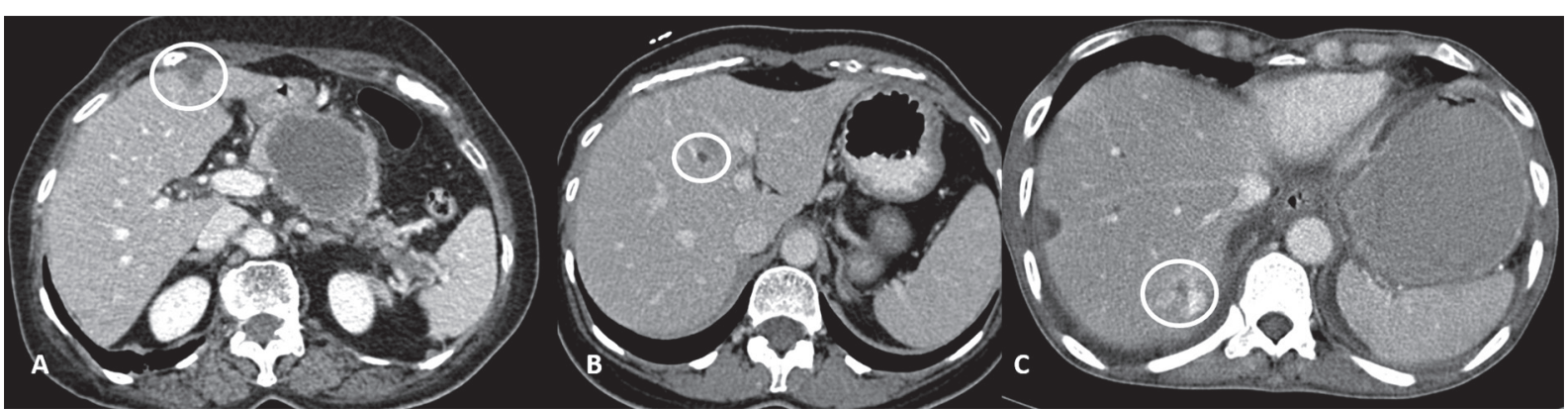

Fig. 8 While considering target lesions, foci of focal fatty infiltration (A), simple cysts (B), and hemangiomas (C) should be carefully assessed and excluded. 


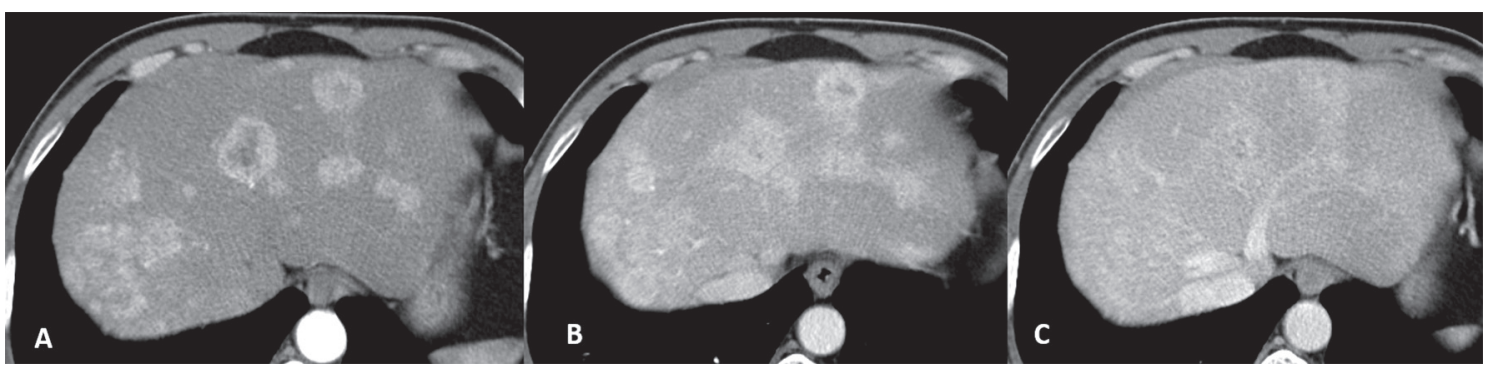

Fig. 9 Multiple arterial enhancing hepatic metastases in a patient with neuroendocrine tumor of the stomach. The lesions in both lobes of the liver become less conspicuous from arterial (A) through portal (B) and venous (C) phases. Thus, follow-up imaging should incorporate a similar protocol, and the lesions should be assessed in the phase where they are best visible.

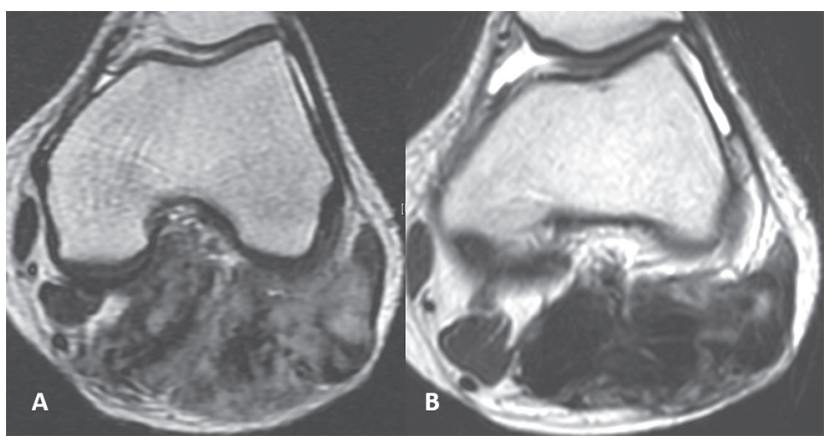

Fig. 10 The patient with fibromatosis (desmoid) in the right popliteal region (A) pre- and (B) postchemotherapy. The lesion shows $<30 \%$ decrease in size at follow-up (B), which would be labeled as stable disease (SD) as per RECIST criteria. However, the heterogeneous T2 hyperintense signal of the tumor has become T2 hypointense on the follow-up scan, indicating fibrotic change in the tumor, which indicates response.

scans including timing of various phases of contrast administration to allow for accurate reproducible measurements.

Furthermore, although an improvement over RECIST 1.0, RECIST 1.1 does have its own set of shortcomings. The roles of PET-CT and MRI remain limited and not well defined. Furthermore, the criteria remain predominantly size based, with changes in tumor morphology not accounted for. For example, tumors such as desmoid often respond to treatment with development of fibrosis and without significant decrease in size. This manifests as the development of T2 hypointense signal on MRI, but this cannot be labeled as response as per RECIST criteria ( Fig. 10). ${ }^{18}$ Similarly, tumors may respond to targeted therapy with decreased attenuation without a decrease in size, which again cannot be labeled as response as per RECIST, leading to the development of alternate response criteria such as Choi criteria (discussed in the next article in detail). Use of immunomodulator drugs may also lead to the development of a new lesion (considered progressive disease by RECIST), which can still be seen in responding patients on these drugs; dedicated tumor response criteria have been developed to take this into account (discussed in the other article). Again, atypical responses such as a paradoxical increase in tumor size due to hemorrhage or necrosis, or the development of cavitation in a lung nodule, cannot be analyzed differently using RECIST. ${ }^{19}$ A recent study by Kuhl et $\mathrm{al}^{20}$ prospectively assessed the validity of RECIST version 1.1 in patients with metastatic disease on systemic chemotherapy. They demonstrated that different cancer sites may appear equally suitable to be selected as target lesions, leading to conflicting response classification with RECIST 1.1. ${ }^{2}$ Thus, the current criteria for target lesions may not reflect accurately overall tumor burden or response.

\section{Take-Home Points for the Practicing Radiologist}

In this article, we have discussed the salient features in tumor response evaluation using RECIST 1.1 criteria. These criteria clarify a lot of basic principles and concepts vis-à-vis assessing tumor response in patients with advanced cancer. At the risk of repetition, it must be emphasized that the criteria are meant only for clinical trials and should not be blanketly used in regular clinical practice. However, the principles behind these criteria must be used while reporting each and every cancer study, merging with routine clinical reporting. It must be borne in mind that referring oncologists are now aware of RECIST 1.1 lexicon, and terms such as "progression" mean a very specific entity to them; hence, such terms should be preferably avoided unless the findings are unequivocal (such as the appearance of multiple new pulmonary metastases). We otherwise prefer using alternative terms conveying the information appropriately (such as increased/decreased disease burden). This gives the referring oncologist the flexibility to consider the radiologic findings and the patient's clinical condition as a whole and make appropriate management decisions.

1. It is not necessary to measure and report details of too many lesions in a patient with widespread metastatic disease, and only a few select lesions should be reported. Also, one is not expected to give sums of longest diameters in routine reports.

2. Most importantly, a small increase in a lesion size by a few millimeters should not be called as progressive disease; the patient should be labeled as having relatively stable tumor burden. If the radiologist sees uniform minimal increase in the size of multiple lesions, he/she can report it as "stable to minimally increased disease burden" or words to that effect. The word "disease progression" should ideally be avoided, and a short-interval follow-up scan may be recommended. In such scenarios, the referring oncologist usually then considers other factors as well before taking a management decision. If the patient is otherwise symptomatically good with relatively stable tumor markers, 
they chemotherapy is usually continued. On the other hand, if the patient is clinically deteriorating or the tumor markers have increased substantially, the chemotherapy may be changed.

3. Similarly, mild increase in ascites or effusion or the appearance of a 3-mm newly apparent pulmonary nodule should not be labeled as worsening disease, particularly if the rest of the disease is relatively stable.

4. Short-axis dimensions of a lymph node are important. Thus, a $13 \times 11 \mathrm{~mm}$ node that now becomes $16 \times 12$ should be considered stable.

5. While it would make things more convenient, the general practice in most institutes (including ours) is to refrain from reporting tumor measurements in a single dimension in routine clinical practice. Bidimensional measurements on the axial plane should be provided for the primary tumor and the visceral and nodal metastases. Providing the craniocaudal dimension is not required in most cases of metastatic disease.

6. It is important to look at all relevant prior scans while reporting the current scan. Often, a slowly growing tumor may not show significant increase from the immediate prior but may demonstrate gradually enlarging metastases when compared with two or three prior studies. As progression is based on increase from the nadir, one must make it a point to have a glance at older scans as well.

In conclusion, we have summarized the history of development of objective tumor response criteria and discussed the RECIST criteria in detail. In-depth knowledge of RECIST and the principles behind it are essential for all practicing radiologists, particularly given the increasing cancer incidence and increasing use of imaging in all cancer patients. Being predominantly size-based criteria, there are limitations in assessing tumor response using RECIST criteria, which should be recognized. This has led to the emergence of various alternative response criteria that are discussed in detail in the subsequent article.

\section{Conflict of Interest}

None declared.

\section{Acknowledgment}

The authors thank Dr. Nilendu Purandare, Tata Memorial Hospital, New Delhi, for providing his guidance and expertise in the writing and review of the manuscript.

\section{References}

1 Therasse P, Arbuck SG, Eisenhauer EA, et al. New guidelines to evaluate the response to treatment in solid tumors. European Organization for Research and Treatment of Cancer, National
Cancer Institute of the United States, National Cancer Institute of Canada. J Natl Cancer Inst 2000;92(3):205-216

2 Eisenhauer EA, Therasse P, Bogaerts J, et al. New response evaluation criteria in solid tumours: revised RECIST guideline (version 1.1) Eur J Cancer 2009;45(2):228-247

3 Choi H. Response evaluation of gastrointestinal stromal tumors. Oncologist 2008;13(Suppl 2):4-7

4 Seymour L, Bogaerts J, Perrone A, et al; RECIST working group. iRECIST: guidelines for response criteria for use in trials testing immunotherapeutics. Lancet Oncol 2017;18(3):e143-e152

5 Hamaoka T, Madewell JE, Podoloff DA, Hortobagyi GN, Ueno NT. Bone imaging in metastatic breast cancer. J Clin Oncol 2004;22(14):2942-2953

6 WHO Handbook for Reporting Results of Cancer Treatment. Vol. 48, World Health Organization Offset Publication; 1979

7 Miller AB, Hoogstraten B, Staquet M, Winkler A. Reporting results of cancer treatment. Cancer 1981;47(1):207-214

8 Moertel CG, Hanley JA. The effect of measuring error on the results of therapeutic trials in advanced cancer. Cancer 1976;38(1):388-394

9 Jaffe CC. Measures of response: RECIST, WHO, and new alternatives. J Clin Oncol 2006;24(20):3245-3251

10 James K, Eisenhauer E, Christian M, et al. Measuring response in solid tumors: unidimensional versus bidimensional measurement. J Natl Cancer Inst 1999;91(6):523-528

11 Rezai P, Pisaneschi MJ, Feng C, Yaghmai V. A radiologist's guide to treatment response criteria in oncologic imaging: anatomic imaging biomarkers. AJR Am J Roentgenol 2013;201(2):237-245

12 Bogaerts J, Ford R, Sargent D, et al; RECIST Working Party. Individual patient data analysis to assess modifications to the RECIST criteria. Eur J Cancer 2009;45(2):248-260

13 Tirkes T, Hollar MA, Tann M, Kohli MD, Akisik F, Sandrasegaran K. Response criteria in oncologic imaging: review of traditional and new criteria. Radiographics 2013;33(5):1323-1341

14 Subbiah V, Chuang HH, Gambhire D, Kairemo K. Defining clinical response criteria and early response criteria for precision oncology: current state-of-the-art and future perspectives. Diagnostics (Basel) 2017;7(1):10

15 Schwartz LH, Bogaerts J, Ford R, et al. Evaluation of lymph nodes with RECIST 1.1. Eur J Cancer 2009;45(2):261-267

16 Schwartz LH, Seymour L, Litière S, et al. RECIST 1.1standardisation and disease-specific adaptations: perspectives from the RECIST Working Group. Eur J Cancer 2016;62 (March):138-145

17 Abramson RG, McGhee CR, Lakomkin N, Arteaga CL. Pitfalls in RECIST data extraction for clinical trials: beyond the basics. Acad Radiol 2015;22(6):779-786

18 Sheth PJ, Del Moral S, Wilky BA, et al. Desmoid fibromatosis: MRI features of response to systemic therapy. Skeletal Radiol 2016;45(10):1365-1373

19 Nishino M, Jagannathan JP, Ramaiya NH, Van den Abbeele AD. Revised RECIST guideline version 1.1: what oncologists want to know and what radiologists need to know. Am J Roentgenol 2010;195(2):281-289

20 Kuhl CK, Alparslan Y, Schmoee J, et al. Validity of RECIST version 1.1 for response assessment in metastatic cancer: a prospective, multireader study. Radiology 2019;290(2): 349-356 